\title{
Thermal Analysis of Mixed Matrix Membranes Involved in Gas Separation
}

\author{
Andrew Paul Han, Frederick F Stewart, \\ Christopher J Orme \\ July 2019
}

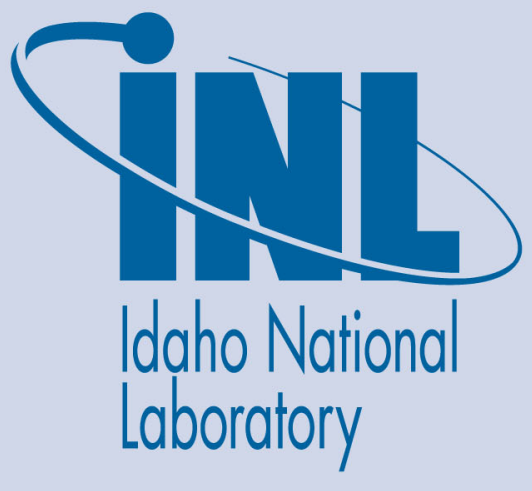

The INL is a U.S. Department of Energy National Laboratory operated by Battelle Energy Alliance 


\title{
Thermal Analysis of Mixed Matrix Membranes Involved in Gas Separation
}

\author{
Andrew Paul Han, Frederick F Stewart, Christopher J Orme
}

July 2019

Idaho National Laboratory Idaho Falls, Idaho 83415

http://www.inl.gov

Prepared for the U.S. Department of Energy Office of Nuclear Energy Under DOE Idaho Operations Office

Contract DE-AC07-05ID14517 


\title{
Thermal Analysis of Mixed Matrix Membranes Involved in Gas Separation
}

\author{
Andrew Han
}

Intern, Biological and Chemical Science and Engineering, Energy and Environment Science and Technology

\section{Objectives}

- Develop the method for forming a mixed matrix membrane that can separate oxygen from air.

- The membrane consists of a polysulfone (PSF) support layer, a selective layer comprising PSF loaded with nanodiamond particles, and if necessary, a polydimethylsiloxane gutter layer to patch defects that often occur during fabrication.

- Separation produces a 90-95\% oxygen rich stream.

- Oxygen from the separation would be used as an oxidant for gasification processes and clean coal combustion.

- Examine how nanodiamonds affect the permeability and selectivity for oxygen in membranes.

- Determine which solvent can yield a membrane with the greatest permeability and ease of formation and solvent removal.

\section{Methods}

- Thermomechanical Analysis (TMA) examines how nanodiamonds affect the polymer's structure by measuring its dimensional change when heated.

- Thermogravimetric Analysis (TGA) verifies the amount of solvent that remains on the polymer once cast by measuring the weight of the polymer as a function of temperature. TGA is also used to determine the upper temperature stability of the membranes.

- Differential Scanning Calorimetry (DSC) evaluates the glass transition temperature, which can be influenced by contamination, such as solvent residues. Residual solvent lowers the glass transition temperature of the polymer.

\section{Images and Data}

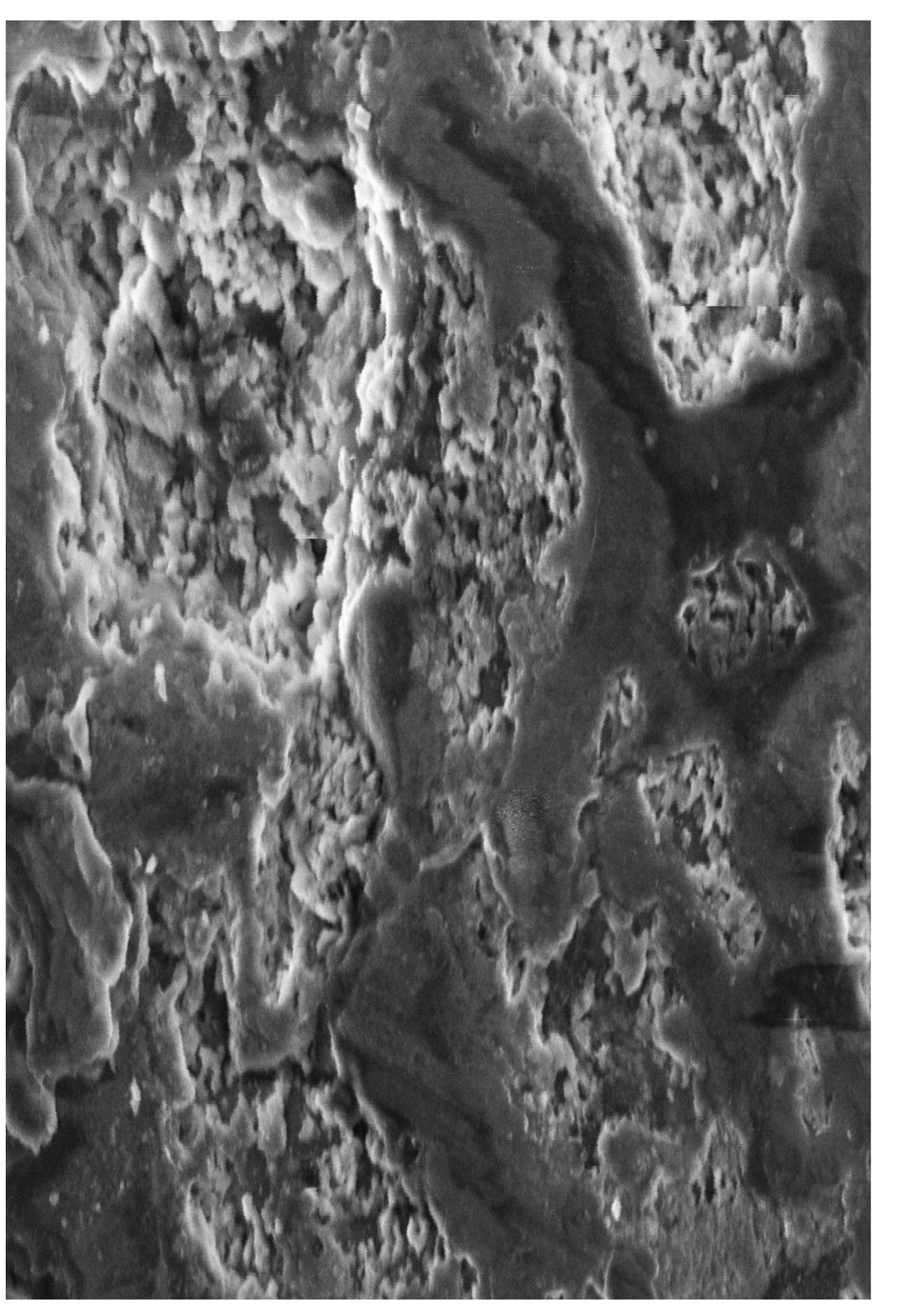

SEM image of UDEL 1700 THF $16 \%$ C18

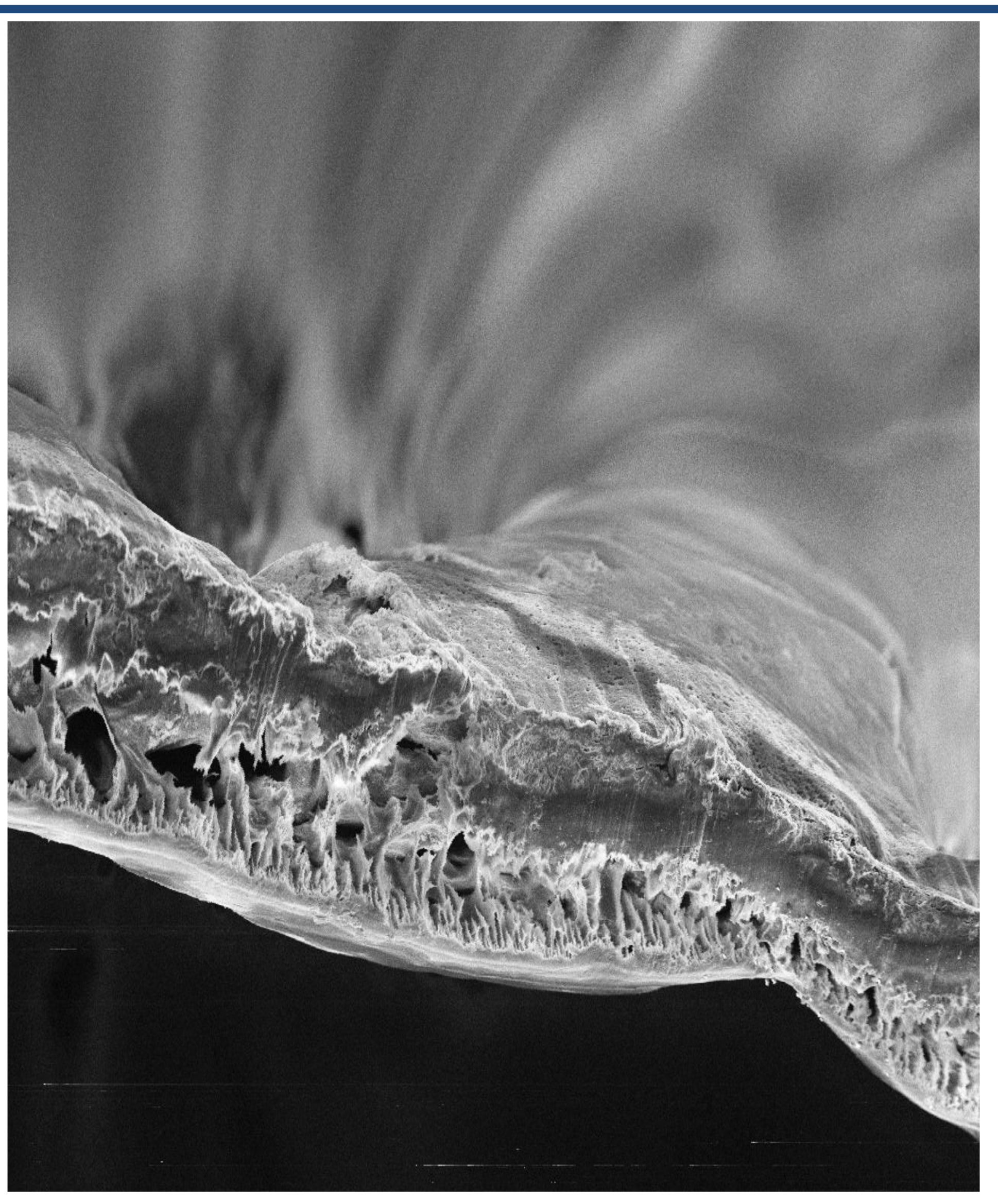

SEM image of Phase inverted UDEL 1700 THF 2\% ND
TGA of UDEL 1700 NMP $0 \%$ ND

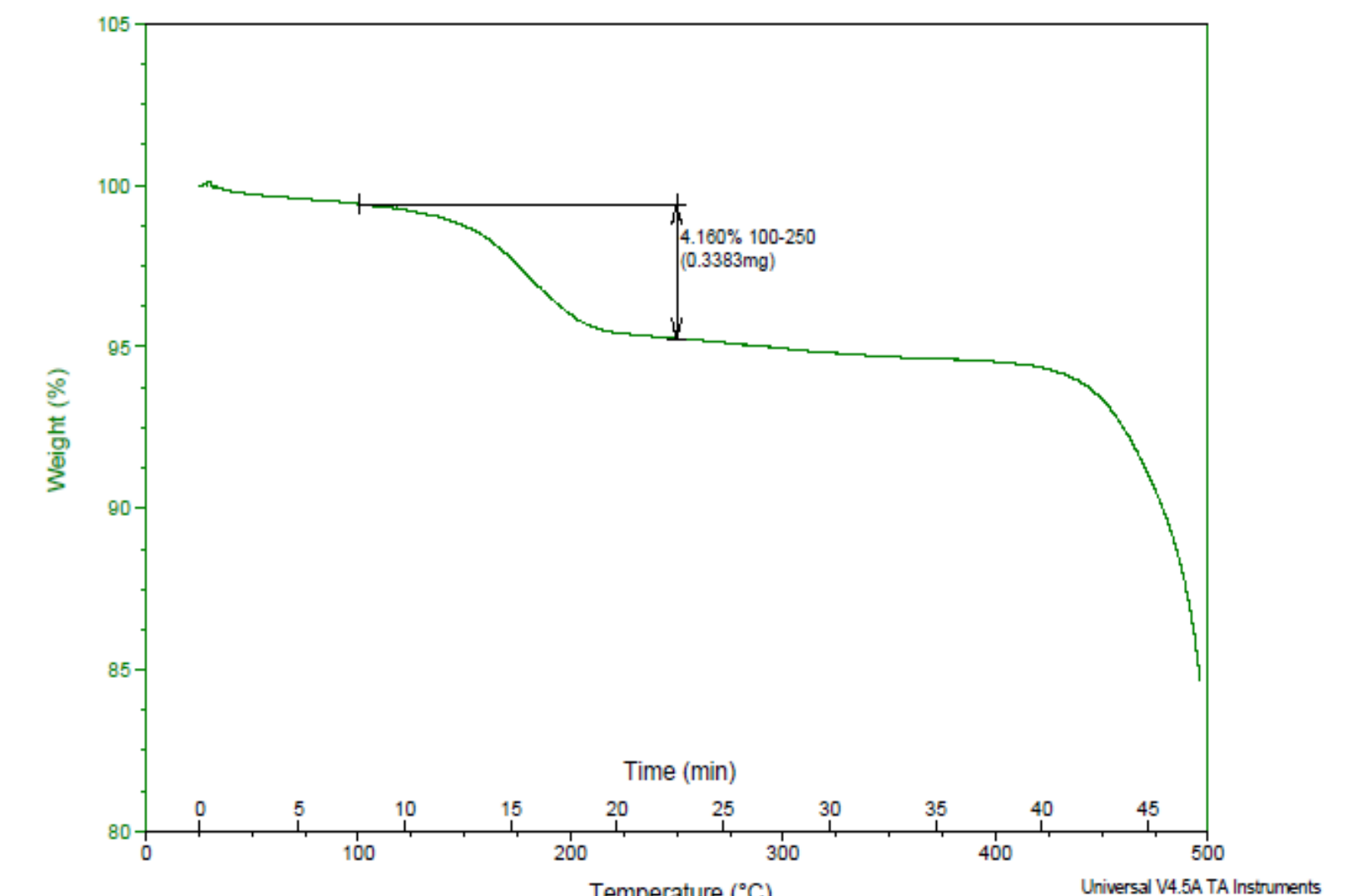

TGA of UDEL 1700 THF $5 \%$ ND

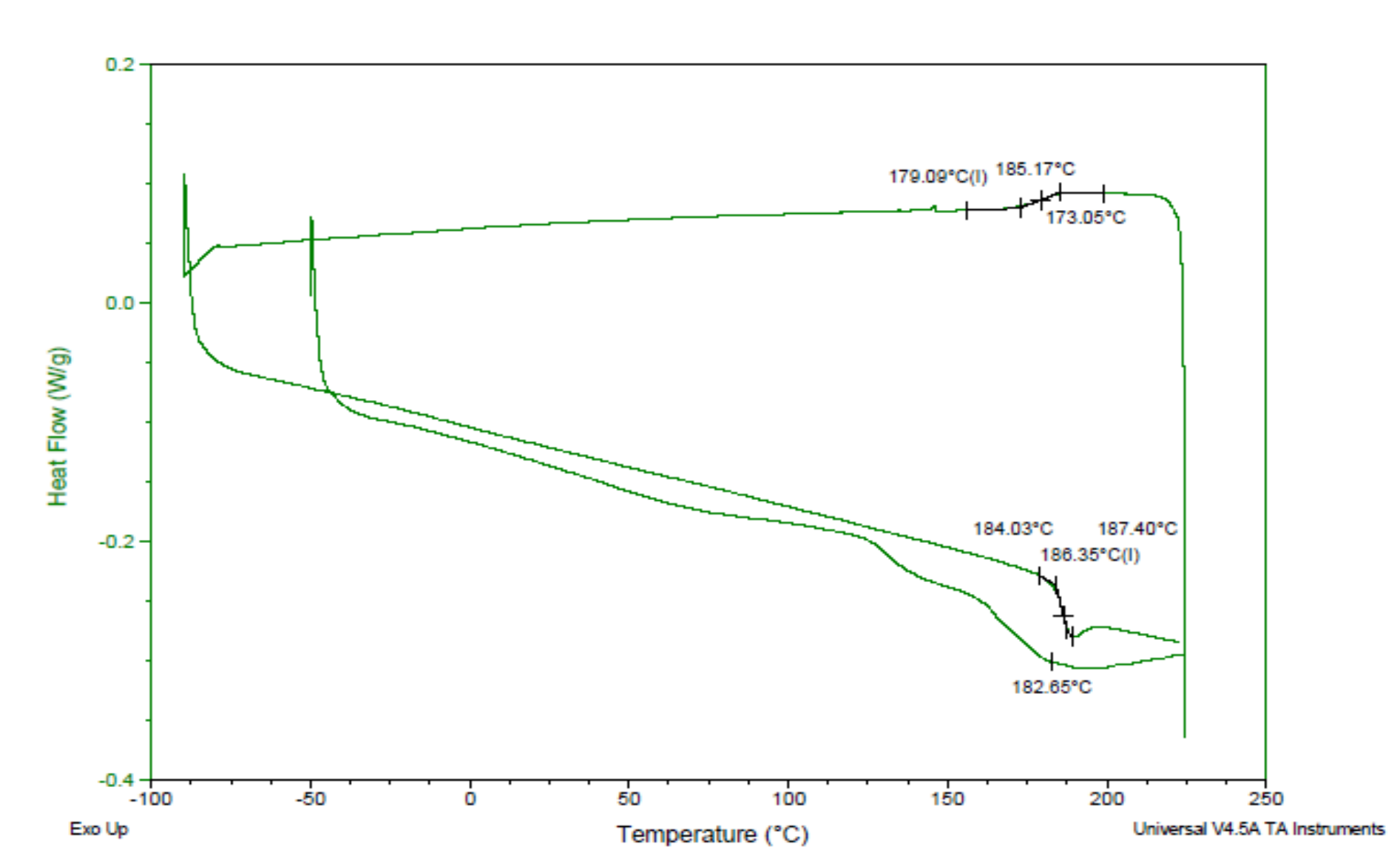

DSC of UDEL 1700 THF $5 \%$ ND

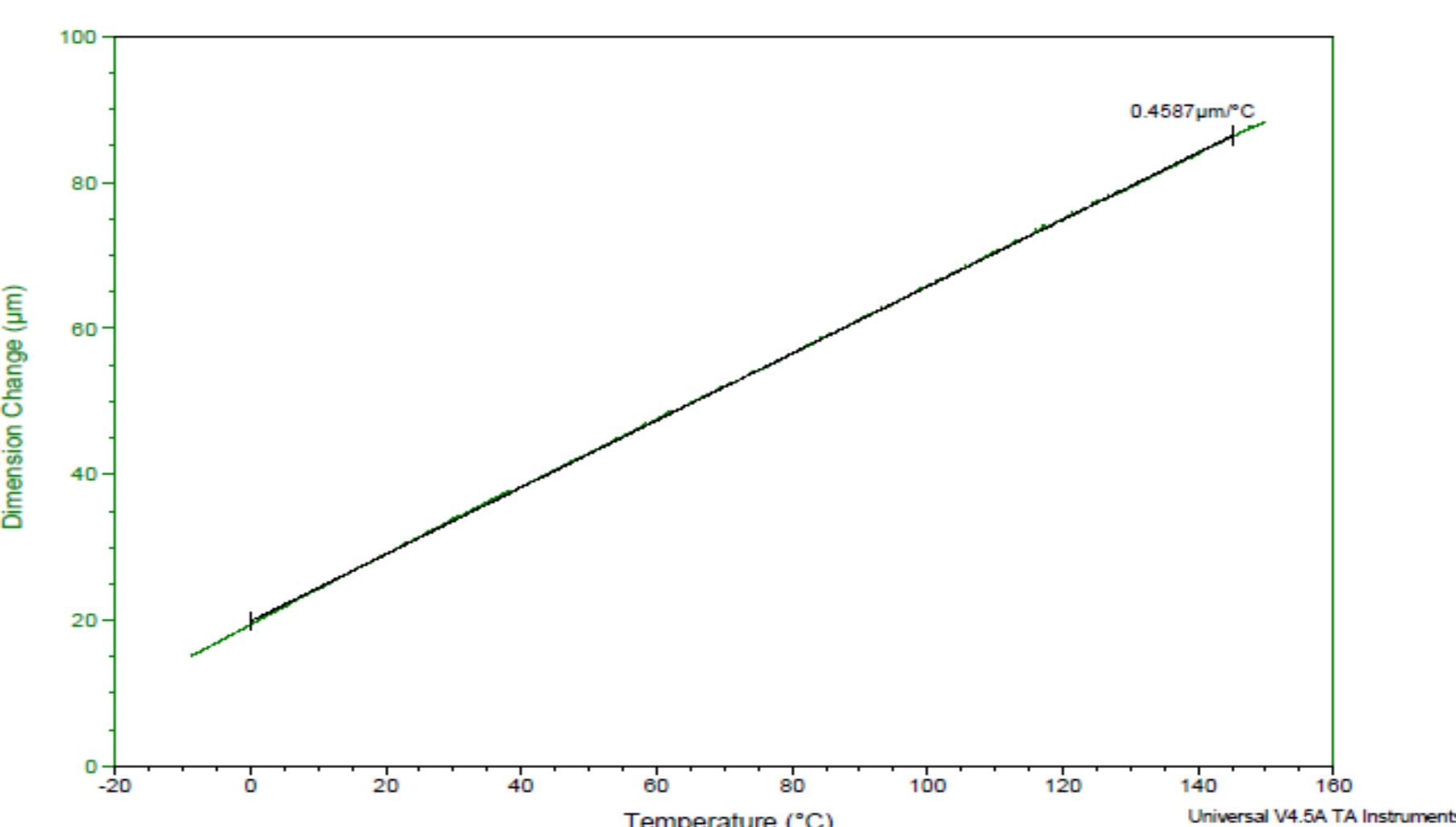

TMA of DND $1 \%$ ND (50nm)

\section{Results and Conclusions}

- As more nanodiamonds (NDs) are placed in the polymer, the expansion rate increases, which shows that NDs can influence the membrane morphology.

- Polysulfone cast from dimethylacetamide (DMAc) or from tetrahydrofuran (THF), if loaded with NDs, were the membranes that had the least amount of weight change as a function of temperature, thus NDs may have aided in the removal of solvent. Polymer membranes cast from these solvents were shown to exhibit the greatest permeability.

- Polysulfone cast in N-methylpyrrolidone (NMP) had the lowest glass transition temperature which suggested that this polymer had the most amount of solvent when cast (this conclusion was supported by TGA). NMP also remained in the polymer at $350-400^{\circ} \mathrm{C}$, well above its boiling point.

- In summary, a method for the formation of solvent-free membranes for separation of oxygen and nitrogen was developed in this work.

\section{Acknowledgements}

I would like to thank Dr. Frederick Stewart and Mr. Christopher Orme for their guidance and support during this project.

TA instruments provided the equipment needed to perform the experiments 


\section{Abstract}

- Idaho National Laboratory is developing a mixed matrix membrane that can separate oxygen from air. The enriched oxygen stream is intended to be used in gasification systems and coal combustion. This study evaluated the thermal properties of polymer membranes that were cast from different solvents and contained various concentrations of nanodiamonds, where the polymer-nanodiamond composites acted as the selective layer. Measuring these properties helped to understand the membrane formation process to yield membranes that give reproducible performance. Thermomechanical Analysis determined that nanodiamonds cause a physical effect on the membrane when exposed to heating. Thermogravimetric Analysis and Differential Scanning Calorimetry determined residual solvent in the membrane after formation. Residual solvent tended to decrease the performance of membranes. From this work, we developed a method of forming solvent-free polysulfone membrane composites containing nanodiamond particles that gave reproducible permeability and selectivity data. 